PROCEEDINGS OF THE

AMERICAN MATHEMATICAL SOCIETY

Volume 126, Number 8, August 1998, Pages 2231-2240

S $0002-9939(98) 04724-8$

\title{
ON THE GRAPH CONVERGENCE OF SUBDIFFERENTIALS OF CONVEX FUNCTIONS
}

\author{
C. COMBARI AND L. THIBAULT
}

(Communicated by Dale Alspach)

\begin{abstract}
This paper provides another proof of the Attouch Theorem relating the epigraphical limit of sequences of convex functions to the set limit of the graphs of the subdifferentials.
\end{abstract}

\section{INTRODUCTION}

Attouch ([1] and [2]) proved that a sequence of proper lower semicontinuous convex functions on a reflexive Banach space Mosco-converges if and only if the graphs of the subdifferentials Painlevé-Kuratowski converge to the graph of the subdifferential of the Mosco-limit function and a condition that fixes the constant of integration (normalization condition) holds. Attouch and Beer generalized in [3] the result in the setting of any Banach space. They showed that in any Banach space slice convergence for proper lower semicontinuous convex functions is equivalent to Painlevé-Kuratowski convergence of the graphs of the subdifferentials plus a normalization condition. Both proofs given in Attouch [1] and Attouch and Beer [3] depend heavily on the integration theorem by Rockafellar [16] using the cyclic monotonicity of the subdifferential of convex functions. These theorems have applications in several fields like convergence problems in mechanics (see, e.g., [2]), evolution equations governed by subdifferential operators (see, e.g., [2] and [4]), numerical optimization (see, e.g., [10]), and generalized second order derivatives of convex functions (see, e.g., [11], [15] and [18]).

The aim of this paper is to provide another proof of the above theorems without using the above integration theorem by Rockafellar. This proof consists in proving in a direct way that under a normalization condition, convergence of the graphs of subdifferentials of lower semicontinuous convex functions $f_{n}$ is equivalent to Mosco-convergence of these functions and their Fenchel conjugates. The key to this approach is the study of the behaviour of limits of strongly convergent sequences of elements in the graphs of subdifferentials with respect to the epigraphical limits superior and inferior of $\left(f_{n}\right)$. This has been done with the generalized Fenchel subdifferential of the epigraphical limit inferior of $\left(f_{n}\right)$ since this function may be nonconvex although all the functions $f_{n}$ are convex. A modification of this approach

Received by the editors January 5, 1996

1991 Mathematics Subject Classification. Primary 49J52, 58C20, 90C25.

Key words and phrases. Epilimit inferior, epilimit superior, Mosco-convergence, slice convergence, Painlevé-Kuratowski convergence. 
will be used in [9] to study the convergence of approximate subdifferentials of convex functions.

\section{Graph CONVERGEnCE of SUbdifferentials}

We begin by recalling the graph convergence theorem of subdifferentials of convex functions proved by Attouch ([1] and [2]) for reflexive spaces.

2.1. Theorem (Attouch). Let $X$ be a reflexive Banach space and let $f, f_{n}: X \rightarrow$ $\mathbb{R} \cup\{+\infty\}, n \in \mathbb{N}$, be proper lower semicontinuous convex functions. Then the following assertions are equivalent:

a) $f_{n} \stackrel{M}{\rightarrow} f$

b)

$$
\left\{\begin{array}{l}
\partial f_{n} \stackrel{P . K}{\longrightarrow} \partial f \\
(N . C): \text { there exist }\left(a, a^{*}\right) \in \partial f \text { and a sequence }\left(a_{n}, a_{n}^{*}\right) \in \partial f_{n} \text { such that } \\
\left(a_{n}, a_{n}^{*}, f_{n}\left(a_{n}\right)\right) \rightarrow\left(a, a^{*}, f(a)\right) .
\end{array}\right.
$$

(Here N.C. stands for the so-called normalization condition.)

We now make precise the notions which have been used. Let $\left(f_{n}\right)$ be a sequence of functions from a topological space $(X, \tau)$ into $\mathbb{R} \cup\{-\infty,+\infty\}$. The sequential epilimit inferior of the sequence $\left(f_{n}\right)$ with respect to the topology $\tau$ is the function denoted by $\tau-L i_{e} f_{n}$ and defined by

$$
\left(\tau-L i_{e} f_{n}\right)(x)=\inf _{x_{n} \rightarrow x} \liminf _{n} f_{n}\left(x_{n}\right) .
$$

The sequential epilimit superior is denoted by $\tau-L s_{e} f_{n}$ and defined by

$$
\left(\tau-L s_{e} f_{n}\right)(x)=\inf _{x_{n} \rightarrow x} \limsup _{n} f_{n}\left(x_{n}\right) .
$$

If $X$ is a normed vector space, one says that the sequence $\left(f_{n}\right)$ Mosco-converges to $f\left(f_{n} \stackrel{M}{\rightarrow} f\right)$ provided

$$
w-L i_{e} f_{n} \geq f \geq s-L s_{e} f_{n}
$$

where $w$ (resp. $s$ ) denotes the weak (resp. strong) topology of $X$. If $\left(g_{n}\right)$ and $g$ are functions defined on the topological dual $X^{*}$ of $X$, one says that $\left(g_{n}\right)^{*}$-Moscoconverges to $g\left(g_{n} \stackrel{M^{*}}{\rightarrow} g\right)$ provided

$$
w^{*}-L i_{e} g_{n} \geq g \geq s-L s_{e} g_{n}
$$

where $w^{*}$ denotes the weak star topology of $X^{*}$.

Recall also that for a sequence $\left(C_{n}\right)$ of subsets of $X$ the sequential limits inferior and superior of $\left(C_{n}\right)$ with respect to the topology $\tau$ are defined by

$$
\tau-L i C_{n}=\left\{x \in X: \exists x_{n} \in C_{n}, x_{n} \stackrel{\tau}{\rightarrow} x\right\}
$$

and

$$
\tau-L s C_{n}=\left\{x \in X: \exists s(n), \exists x_{s(n)} \in C_{s(n)}, x_{s(n)} \stackrel{\tau}{\rightarrow} x\right\} .
$$

The sequence $\left(C_{n}\right)$ is said to be Painlevé-Kuratowski convergent to $C\left(C_{n} \stackrel{P . K .}{\rightarrow} C\right)$ provided

$$
\tau-L s C_{n} \sqsubset C \sqsubset \tau-L i C_{n} .
$$


If $X$ is a normed space and $f: X \rightarrow \mathbb{R} \cup\{+\infty\}$ is convex, one denotes by $\partial f$ the graph of the subdifferential of $f$ :

$$
\begin{aligned}
\partial f & =\left\{\left(x, x^{*}\right) \in X \times X^{*}: x^{*} \in \partial f(x)\right\} \\
& =\left\{\left(x, x^{*}\right) \in X \times X^{*}: f(x)+f^{*}\left(x^{*}\right)=\left\langle x^{*}, x\right\rangle\right\}
\end{aligned}
$$

where $f^{*}$ is the Fenchel conjugate of $f$, that is, $f^{*}\left(x^{*}\right)=\sup \left\{\left\langle x^{*}, x\right\rangle-f(x): x \in\right.$ $X\}$. We will also denote by $\partial^{*} f^{*}$ the graph of the subdifferential of $f^{*}$ restricted to $X^{*} \times X$ (and not $X^{*} \times X^{* *}$ ). So $x \in \partial^{*} f^{*}\left(x^{*}\right)$ is equivalent to $x^{*} \in \partial f(x)$.

When $\varphi: X \rightarrow \mathbb{R} \cup\{-\infty,+\infty\}$ is a nonnecessarily convex function one may extend the above definition by considering the Fenchel subdifferential notion. Recall that the Fenchel subdifferential of $\varphi$ is the subset given by

$$
\partial^{F e n} \varphi(x)=\left\{x^{*} \in X^{*}:\left\langle x^{*}, u-x\right\rangle+\varphi(x) \leq \varphi(u), \forall u \in X\right\}
$$

if $x \in \operatorname{dom} \varphi:=\{x \in X: \varphi(x)<+\infty\}$, and by $\partial^{F e n} \varphi(x)=\varnothing$ if $x \notin \operatorname{dom} \varphi$.

Recall also that $\varphi$ is said to be proper provided $\operatorname{dom} \varphi \neq \varnothing$ and $\varphi(x)>-\infty$ for every $x \in X$.

Let us now point out that the proofs of the graph convergence theorem given by Attouch ([1] and [2]) and Attouch and Beer [3] depend heavily on the following result by Rockafellar ([16] and [17]) (as well as its dual version in [3]).

2.2. Theorem (Rockafellar). Let $X$ be a Banach space and $f: X \rightarrow \mathbb{R} \cup\{+\infty\}$ be a proper lower semicontinuous convex function. For each $x \in X$ and $x_{0} \in$ $\operatorname{dom} \partial f:=\{u \in X: \partial f(u) \neq \varnothing\}$

$$
f(x)=\sup \left\{f\left(x_{0}\right)+\sum_{i=1}^{n-1}\left\langle x_{i}^{*}, x_{i+1}-x_{i}\right\rangle\right\}
$$

where the supremum is taken over all chains $x_{1}=x_{0}, x_{2}, \ldots, x_{n}=x$, where the intermediate points lie in $\operatorname{dom} \partial f$, and where $x_{i}^{*} \in \partial f\left(x_{i}\right)$.

We are going to use another integration result and give another proof (different to those by Attouch [1] and Attouch and Beer [3]) for the theorem relating the graph convergence of subdifferentials to some specific convergence (see [2] and [5]) of the functions.

In all the sequel $X$ will be any Banach space.

We start with the following lemma whose proof may be deduced from Rockafellar's theorem [16]. For the convenience of the reader, we will give a direct proof in the appendix by adapting Rockafellar's proof (where both functions $f$ and $g$ are assumed to be convex) to the case below.

2.3. Lemma. Let $g: X \rightarrow \mathbb{R} \cup\{+\infty\}$ be a lower semicontinuous function and $f: X \rightarrow \mathbb{R} \cup\{+\infty\}$ be a proper lower semicontinuous convex function. If $\partial f \sqsubset$ $\partial^{F e n} g$, then $f=g+$ Const (where Const denotes a constant real number).

Before giving our proof of the graph convergence theorem of subdifferentials we need to consider another lemma.

2.4. Lemma. Let $f_{n}: X \rightarrow \mathbb{R} \cup\{+\infty\}, n \in \mathbb{N}$, be proper lower semicontinuous convex functions satisfying the hypothesis

$(\mathrm{H}): \quad s-L i_{e} f_{n}>-\infty$ and there exists a strongly convergent sequence $\left(a_{n}\right)$ for which

$$
\limsup _{n} f_{n}\left(a_{n}\right)<+\infty
$$


Then for any sequence $\left(x_{n}, x_{n}^{*}\right)$ with $\left(x_{n}, x_{n}^{*}\right) \in \partial f_{n}$ and $\left(x_{n}, x_{n}^{*}\right) \stackrel{\|\cdot\| x\|\cdot\|_{*}}{\longrightarrow}\left(x, x^{*}\right)$ one has

a) $\left(w-L i_{e} f_{n}\right)(x)=\liminf _{n} f_{n}\left(x_{n}\right)$ and $\left(s-L s_{e} f_{n}\right)(x)=\limsup f_{n}\left(x_{n}\right)$;

b) $\left(x, x^{*}\right) \in \partial^{F e n}\left(w-L i_{e} f_{n}\right) \cap \partial\left(s-L s_{e} f_{n}\right)$.

So under hypothesis $(\mathrm{H})$ we have

$$
\operatorname{Li\partial f}_{n} \sqsubset \partial^{F e n}\left(w-L i_{e} f_{n}\right) \cap \partial\left(s-L s_{e} f_{n}\right) .
$$

Moreover, the same results hold if the functions $f_{n}$ are defined on $X^{*}$ and $w-L i_{e} f_{n}$ is replaced by $w^{*}-L i_{e} f_{n}$.

Remark. We consider the Fenchel subdifferential since the function $w-L i_{e} f_{n}$ need not be convex although the functions $f_{n}$ are convex.

Proof of Lemma 2.4. Fix a sequence $\left(x_{n}, x_{n}^{*}\right) \in \partial f_{n}$ strongly converging to $\left(x, x^{*}\right)$ in $X \times X^{*}$. For $y \in X$ and $y_{n} \stackrel{w}{\rightarrow} y$ one has

$$
\left\langle x_{n}^{*}, y_{n}-x_{n}\right\rangle+f_{n}\left(x_{n}\right) \leq f_{n}\left(y_{n}\right)
$$

and hence

$$
\left\langle x^{*}, y-x\right\rangle+\liminf _{n} f_{n}\left(x_{n}\right) \leq \liminf _{n} f_{n}\left(y_{n}\right) .
$$

If we take $y=a$ and $y_{n}=a_{n}$ in (1) we get (using (H)) $\liminf _{n} f_{n}\left(x_{n}\right)<+\infty$ and hence by the first part of $(\mathrm{H})$ we have that $\liminf _{n} f_{n}\left(x_{n}\right)$ is finite. Taking in (1) the infimum over all sequences $y_{n} \stackrel{w}{\rightarrow} y$ gives

$$
\left\langle x^{*}, y-x\right\rangle+\liminf _{n} f_{n}\left(x_{n}\right) \leq\left(w-L i_{e} f_{n}\right)(y)
$$

and setting $y=x$ in (2) we obtain

$$
\liminf _{n} f_{n}\left(x_{n}\right) \leq\left(w-L i_{e} f_{n}\right)(x)
$$

which ensures (by definition)

$$
\left(w-L i_{e} f_{n}\right)(x)=\liminf _{n} f_{n}\left(x_{n}\right) .
$$

So it follows from (2) and (3) that $\left(x, x^{*}\right) \in \partial^{F e n}\left(w-L i_{e} f_{n}\right)$ and this proves the first inclusion in b). The proof of the second one is similar and the same arguments still hold for functions $f_{n}$ defined on $X^{*}$.

Following Beer [5] we denote $\Delta \varphi:=\left\{\left(x, \varphi(x), x^{*}\right) \in X \times \mathbb{R} \times X^{*}: x^{*} \in \partial \varphi(x)\right\}$. (Here $\varphi$ is assumed to be a convex function from $X$ into $\mathbb{R} \cup\{+\infty\}$.)

We can now establish our proof of the theorem.

2.5. Theorem. Let $f, f_{n}: X \rightarrow \mathbb{R} \cup\{+\infty\}, n \in \mathbb{N}$, be proper lower semicontinuous convex functions. Then the following assertions are equivalent:

a) $f_{n} \stackrel{M}{\rightarrow} f$ and $f_{n}^{*} \stackrel{M^{*}}{\rightarrow} f^{*}$,

b) $\Delta f_{n} \stackrel{P . K .}{\rightarrow} \Delta f$

c) $\partial f_{n} \stackrel{P . K .}{\rightarrow} \partial f$, and

(N.C.): there exist $\left(a, a^{*}\right) \in \partial f$ and a sequence $\left(a_{n}, a_{n}^{*}\right) \in \partial f_{n}$ such that $\left(a_{n}, a_{n}^{*}, f_{n}\left(a_{n}\right)\right) \rightarrow\left(a, a^{*}, f(a)\right)$. 
Proof. a) $\Rightarrow$ b). For the inclusion $\Delta f \sqsupset L i \Delta f_{n}$ we follow the proof by Attouch [1], [2]. Fix $\left(x, f(x), x^{*}\right) \in \Delta f$. There exist (by properties of Mosco-convergence, see [2] and [6] for example) $u_{n} \stackrel{\|\|}{\rightarrow} x$ and $u_{n}^{*} \stackrel{\|\|_{*}}{\rightarrow} x^{*}$ such that $f_{n}\left(u_{n}\right) \rightarrow f(x)$ and $f_{n}^{*}\left(u_{n}^{*}\right) \rightarrow f^{*}\left(x^{*}\right)$.

Put $\varepsilon_{n}:=f_{n}^{*}\left(u_{n}^{*}\right)+f_{n}\left(u_{n}\right)-\left\langle u_{n}^{*}, u_{n}\right\rangle \geq 0$. Then $\left(\varepsilon_{n}\right)$ converges to $f^{*}\left(x^{*}\right)+$ $f(x)-\left\langle x^{*}, x\right\rangle=0$ and $u_{n}^{*} \in \partial \varepsilon_{n} f_{n}\left(u_{n}\right)$ (the $\varepsilon_{n}$-approximate subdifferential). By the Brondsted-Rockafellar Lemma [8] there exists $\left(x_{n}, x_{n}^{*}\right) \in \partial f_{n}$ such that $\left\|x_{n}-u_{n}\right\| \leq$ $\sqrt{\varepsilon_{n}}$ and $\left\|x_{n}^{*}-u_{n}^{*}\right\| \leq \sqrt{\varepsilon_{n}}$. This ensures $\left(x_{n}, x_{n}^{*}\right) \stackrel{\|\cdot\|}{\rightarrow}\left(x, x^{*}\right)$.

Noting now that a) ensures that $(\mathrm{H})$ holds, we obtain by Lemma 2.4

$$
\limsup _{n} f_{n}\left(x_{n}\right)=f(x)=\liminf _{n} f_{n}\left(x_{n}\right)
$$

and hence $\left(x, f(x), x^{*}\right) \in \operatorname{Li} \Delta f_{n}$. So the inclusion is proved.

It remains to show that $L s \Delta f_{n} \sqsubset \Delta f$. Fix now $\left(x, r, x^{*}\right) \in L s \Delta f_{n}$ and consider a subsequence $\left(x_{k}, f_{k}\left(x_{k}\right), x_{k}^{*}\right) \rightarrow\left(x, r, x^{*}\right)$ with $x_{k}^{*} \in \partial f_{k}\left(x_{k}\right)$ (here $k \in K \subset \mathbb{N}$ ). By Lemma 2.4 one has $\left(x, x^{*}\right) \in \partial f$ and $f_{k}\left(x_{k}\right) \rightarrow f(x)$ and hence $r=f(x)$ and $\left(x, r, x^{*}\right) \in \Delta f$. This completes the proof of $\left.\left.\mathrm{a}\right) \Rightarrow \mathrm{b}\right)$.

$\mathrm{b}) \Rightarrow \mathrm{c})$. It is obvious that b) ensures that $\partial f \sqsubset \operatorname{Li} \partial f_{n}$. Fix now $\left(x, x^{*}\right) \in \operatorname{Ls} \partial f_{n}$, and choose a subsequence $\left(x_{k}, x_{k}^{*}\right) \in \partial f_{k}$ for $k \in K \subset \mathbb{N}$ with $\left(x_{k}, x_{k}^{*}\right) \rightarrow\left(x, x^{*}\right)$. Taking another subsequence if necessary we may suppose by Lemma $2.4 f_{k}\left(x_{k}\right) \rightarrow$ $r \in \mathbb{R}$. So by the assumption we get $\left(x, r, x^{*}\right) \in \Delta f$ and hence $\left(x, x^{*}\right) \in \partial f$. Then we conclude $L s \partial f_{n} \sqsubset \partial f$.

c) $\Rightarrow$ a). It is not difficult to see that the normalization condition (N.C.) ensures the hypothesis $(\mathrm{H})$ in Lemma 2.4. So we have by this lemma

$$
\partial f \sqsubset L i \partial f_{n} \sqsubset \partial^{F e n}\left(w-L i_{e} f_{n}\right) \cap \partial\left(s-L s_{e} f_{n}\right)
$$

and by Lemma 2.3 we may deduce

$$
f=w-L i_{e} f_{n}+C_{1}=s-L s_{e} f_{n}+C_{2}
$$

where $C_{1}$ and $C_{2}$ are two constant real numbers. Moreover, Lemma 2.4 once again and condition (N.C.) ensure the following equality in $\mathbb{R}$ :

$$
\left(w-L i_{e} f_{n}\right)(a)=f(a)=\left(s-L s_{e} f_{n}\right)(a) .
$$

It follows, from (1) and (2), that $C_{1}=C_{2}=0$ and hence (1) becomes $f_{n} \stackrel{M}{\rightarrow} f$. To show $f_{n}^{*} \stackrel{M^{*}}{\rightarrow} f^{*}$ it is enough to note that

$$
\partial f_{n} \stackrel{P . K .}{\rightarrow} \partial f \Leftrightarrow \partial^{*} f_{n}^{*} \stackrel{P . K .}{\rightarrow} \partial^{*} f^{*}
$$

and that (N.C.) ensures the condition (N.C*.):

there exist $\left(a^{*}, a\right) \in \partial^{*} f^{*}$ and a sequence $\left(a_{n}^{*}, a_{n}\right) \in$

$\partial^{*} f_{n}^{*}$ such that $\left(a_{n}^{*}, a_{n}, f_{n}^{*}\left(a_{n}^{*}\right)\right) \rightarrow\left(a^{*}, a, f^{*}\left(a^{*}\right)\right)$,

and to use similar arguments since Lemma 2.3 still holds for functions defined on $X^{*}$ (see the appendix).

This theorem also shows that the Mosco-convergence of $\left(f_{n}\right)$ to $f$ in a nonreflexive Banach space does not ensure the graph convergence of the subdifferential. Indeed in any such space there exist sequences $\left(f_{n}\right)$ that $M$-converge and such that the sequences of Fenchel conjugates $\left(f_{n}^{*}\right)$ do not $M^{*}$-converge (see [7]). However in reflexive spaces, a theorem of Mosco [14] says that the $M$-convergence of proper lower semicontinuous convex functions and that their conjugates are equivalent. So the Attouch theorem (see [1] and [2]) is a direct consequence of Theorem 2.5. 
Before relating Theorem 2.5 to the Attouch-Beer Theorem (see [3]), let us establish the following lemma which also has its own interest. We first need some facts about the notion of slice convergence.

By Theorem 8.2.2 in Beer [6], in the class of proper lower semicontinuous convex functions a sequence $\left(f_{n}\right)_{n}$ slice-converges to $f$ iff for any nonempty open subsets $U$ and $V$ in $X$ and $X^{*}$ respectively and any real number $a>0$ there exists $N$ such that

Epi $f \cap(U \times]-\infty, \alpha[) \neq \varnothing \Rightarrow \operatorname{Epi} f_{n} \cap(U \times]-\infty, \alpha[) \neq \varnothing \quad \forall n \geq N$, Epi $f^{*} \cap(V \times]-\infty, \alpha[) \neq \varnothing \Rightarrow \operatorname{Epif}_{n}^{*} \cap(V \times]-\infty, \alpha[) \neq \varnothing \quad \forall n \geq N$.

2.6. Lemma. Let $f, f_{n}: X \rightarrow \mathbb{R} \cup\{+\infty\}, n \in \mathbb{N}$, be proper lower semicontinuous convex functions. Then the following assertions are equivalent:

a) $f_{n} \stackrel{M}{\rightarrow} f$ and $f_{n}^{*} \stackrel{M^{*}}{\rightarrow} f^{*}$,

b) $\left(f_{n}\right)_{n}$ slice converges to $f$,

c) $f \geq s-L s_{e} f_{n}$ and $f^{*} \geq s-L s_{e} f_{n}^{*}$,

d) for each $x \in X$ and each $x^{*} \in X^{*}$ there exist $x_{n} \stackrel{\|\cdot\|}{\rightarrow} x$ and $x_{n}^{*} \stackrel{\|\cdot\|}{\rightarrow} x^{*}$ such that $f(x) \geq \lim _{n} f_{n}\left(x_{n}\right)$ and $f^{*}\left(x^{*}\right) \geq \lim _{n} f_{n}^{*}\left(x_{n}^{*}\right)$,

e) for each $x \in \operatorname{dom} \partial f$ and each $x^{*} \in \operatorname{dom} \partial^{*} f^{*}$ there exist $x_{n} \stackrel{\|\cdot\|}{\rightarrow} x$ and $x_{n}^{*} \stackrel{\|\cdot\|}{\rightarrow} x^{*}$ such that $f(x) \geq \lim _{n} f_{n}\left(x_{n}\right)$ and $f^{*}\left(x^{*}\right) \geq \lim _{n} f_{n}^{*}\left(x_{n}^{*}\right)$.

Proof. The implications $\mathrm{c}) \Rightarrow \mathrm{d}$ ) and $\mathrm{d}) \Rightarrow \mathrm{e}$ ) are obvious and a) $\Rightarrow$ b) follows easily from the characterization above of slice convergence. So it is enough to prove the implications $\mathrm{e}) \Rightarrow \mathrm{c}), \mathrm{b}) \Rightarrow \mathrm{c}$ ) and $\mathrm{c}) \Rightarrow \mathrm{a}$ ).

e) $\Rightarrow$ c). Assertion e) obviously ensures $f(x) \geq\left(s-L s_{e} f_{n}\right)(x)$ and $f^{*}\left(x^{*}\right) \geq$ $\left(s-L s_{e} f_{n}^{*}\right)\left(x^{*}\right)$ for any $x \in \operatorname{dom} \partial f$ and any $x^{*} \in \operatorname{dom} \partial^{*} f^{*}$. Hence, c) follows from Lemma 3.1 in the appendix.

b) $\Rightarrow \mathrm{c})$. We fix $x \in X$ and we are going to prove $f(x) \geq\left(s-L s_{e} f_{n}\right)(x)$. Obviously we may suppose $r:=f(x) \in \mathbb{R}$. Then for each integer $k \geq 1$ one has Epi $\left.f \cap \mathbb{B}\left(x, \frac{1}{k}\right) \times\right]-\infty, r+\frac{1}{k}[\neq \varnothing$ and hence by the characterization above of slice convergence, one may find an increasing sequence of integers $\left(p_{k}\right)$ such that $p_{k} \geq k$ and elements $\left.\left(u_{n, k}, r_{n, k}\right) \in \operatorname{Epi} f_{n} \cap \mathbb{B}\left(x, \frac{1}{k}\right) \times\right]-\infty, r+\frac{1}{k}$ [ for all $n \geq p_{k}$. If for each $k \geq 1$ and each integer $n \in\left[p_{k}, p_{k+1}\right.$ [ one sets $x_{n}=u_{n, k}$, then one has

$$
f_{n}\left(x_{n}\right) \leq r_{n, k} \leq r+\frac{1}{k}=f(x)+\frac{1}{k} \quad \text { and } \quad x_{n} \in \mathbb{B}\left(x, \frac{1}{k}\right) \text {. }
$$

Therefore $x_{n} \stackrel{\|\cdot\|}{\rightarrow} x$ and $f(x) \geq \lim \sup _{n} f_{n}\left(x_{n}\right) \geq\left(s-L s_{e} f_{n}\right)(x)$.

Similarly we complete the proof to obtain assertion c).

c) $\Rightarrow$ a). We start with the proof of the inequality

$$
w^{*}-L i_{e} f_{n}^{*} \geq\left(s-L s_{e} f_{n}\right)^{*} .
$$

Fix any $x \in X$ and choose $x_{n} \stackrel{\|\cdot\|}{\rightarrow} x$ such that $\left(s-L s_{e} f_{n}\right)(x)=\lim f_{n}\left(x_{n}\right)$. Consider any $x^{*} \in X^{*}$ and $x_{n}^{*} \stackrel{w^{*}}{\rightarrow} x^{*}$. By the Fenchel inequality we have

$$
f_{n}^{*}\left(x_{n}^{*}\right) \geq\left\langle x_{n}^{*}, x_{n}\right\rangle-f_{n}\left(x_{n}\right)
$$

and hence

$$
\liminf _{n} f_{n}^{*}\left(x_{n}^{*}\right) \geq \liminf _{n}\left(\left\langle x_{n}^{*}, x_{n}\right\rangle-f_{n}\left(x_{n}\right)\right)=\left\langle x^{*}, x\right\rangle-\left(s-L s_{e} f_{n}\right)(x) .
$$


Therefore, we have

$$
\begin{aligned}
\left(w^{*}-L i_{e} f_{n}^{*}\right)\left(x^{*}\right) & =\inf _{\substack{w^{*} \\
x_{n}^{*} \rightarrow x^{*}}} \liminf _{n} f_{n}^{*}\left(x_{n}^{*}\right) \\
& \geq \sup _{x \in X}\left(\left\langle x^{*}, x\right\rangle-\left(s-L s_{e} f_{n}\right)(x)\right) \\
& =\left(s-L s_{e} f_{n}\right)^{*}\left(x^{*}\right),
\end{aligned}
$$

which is the desired inequality. Similarly, we have

$$
w-L i_{e} f_{n} \geq\left(s-L s_{e} f_{n}^{*}\right)^{*} .
$$

Now assertion c) and the inequalities (1) and (2) imply

$$
s-L s_{e} f_{n}^{*} \leq f^{*} \leq\left(s-L s_{e} f_{n}\right)^{*} \leq w^{*}-L i_{e} f_{n}^{*}
$$

and

$$
s-L s_{e} f_{n} \leq f \leq\left(s-L s_{e} f_{n}^{*}\right)^{*} \leq w-L i_{e} f_{n}
$$

and hence $f_{n}^{*} \stackrel{M^{*}}{\rightarrow} f^{*}$ and $f_{n} \stackrel{M}{\rightarrow} f$. So the proof is complete.

Remark. The proof of $\mathrm{a}) \Rightarrow \mathrm{b}), \mathrm{b}) \Rightarrow \mathrm{c}$ ) and $\mathrm{c}) \Rightarrow \mathrm{a}$ ) is valid in any normed linear space.

The equivalence $\mathrm{b}$ ) $\Leftrightarrow \mathrm{e}$ ) (from which $\mathrm{c}$ ) and d) follow easily) was already proved by a different method in Attouch-Beer [3].

Now Lemma 2.6 allows us to reformulate Theorem 2.5 with the statement of Attouch-Beer's theorem [3] and to also get a theorem by Beer [5]. The equivalence $\mathrm{a}) \Leftrightarrow \mathrm{c}$ ) is proved by Attouch-Beer [3] and $\mathrm{a}) \Leftrightarrow \mathrm{b}$ ) is similar to Theorem 4.7 in Beer [5].

2.7. Corollary (see [3] and [5]). Let $f, f_{n}: X \rightarrow \mathbb{R} \cup\{+\infty\}, n \in \mathbb{N}$, be proper lower semicontinuous convex functions. Then the following assertions are equivalent:

a) $\left(f_{n}\right)_{n}$ slice converges to $f$,

b) $\Delta f_{n} \stackrel{P . K .}{\rightarrow} \Delta f$

c) $\partial f_{n} \stackrel{P . K .}{\rightarrow} \partial f$, and

(N.C.): there exist $\left(a, a^{*}\right) \in \partial f$ and a sequence $\left(a_{n}, a_{n}^{*}\right) \in \partial f_{n}$ such that $\left(a_{n}, a_{n}^{*}, f_{n}\left(a_{n}\right)\right) \rightarrow\left(a, a^{*}, f(a)\right)$.

\section{Appendix}

In this section we are going to give a direct proof of Lemma 2.3.

3.1. Lemma. Let $g: X \rightarrow \mathbb{R} \cup\{+\infty\}$ and $k: X^{*} \rightarrow \mathbb{R} \cup\{+\infty\}$ be proper lower semicontinuous functions and $f: X \rightarrow \mathbb{R} \cup\{+\infty\}$ be a proper lower semicontinuous convex function. Then

a) $f \geq g$ whenever $f(x) \geq g(x)$ for every $x \in \operatorname{dom} \partial f$;

b) $f^{*} \geq k$ whenever $f^{*}\left(x^{*}\right) \geq k\left(x^{*}\right)$ for every $x^{*} \in \operatorname{dom} \partial^{*} f^{*}$.

Proof. The lemma is a direct consequence of the lower semicontinuity of $g$ and $k$ and of the Brondsted-Rockafellar theorem [8] ensuring for any $x \in \operatorname{dom} f$ and any $x^{*} \in \operatorname{dom} f^{*}$

$$
f(x)=\liminf _{\substack{u \rightarrow x \\ u \in \operatorname{dom} \partial f}} f(u) \quad \text { and } \quad f^{*}\left(x^{*}\right)=\liminf _{\substack{u^{*} \rightarrow x^{*} \\ u^{*} \in \operatorname{dom} \partial^{*} f^{*}}} f^{*}\left(u^{*}\right) .
$$


In the proof of Lemma 3.3 (used in Section 2) we will employ the following result where for any function $\varphi: X^{* *} \rightarrow \mathbb{R} \cup\{+\infty\}$ we denote by $\varphi_{X}$ the restriction of $\varphi$ to $X$.

3.2. Lemma. Let $h: X \rightarrow \mathbb{R} \cup\{+\infty\}$ and $k: X^{*} \rightarrow \mathbb{R} \cup\{+\infty\}$ be proper. Then

a) $x^{*} \in \partial^{F e n} h(x) \Rightarrow h(x)=\left(h_{X}^{* *}\right)(x)$ and $x^{*} \in \partial\left(h_{X}^{* *}\right)(x)$;

b) $x \in \partial^{\text {Fen }} k\left(x^{*}\right) \Rightarrow k\left(x^{*}\right)=\left(k_{X}^{*}\right)^{*}\left(x^{*}\right)$ and $x \in \partial^{*}\left(k_{X}^{*}\right)^{*}\left(x^{*}\right)$.

Moreover, if $h$ and $k$ are lower semicontinuous with respect to the norm and the weak star topology respectively, then

c) $h$ is convex if and only if $\partial\left(h_{X}^{* *}\right) \neq \varnothing$ and $\partial\left(h_{X}^{* *}\right) \sqsubset \partial^{F e n} h$;

d) $k$ is convex if and only if $\partial\left(k_{X}^{*}\right)^{*} \neq \varnothing$ and $\partial^{*}\left(k_{X}^{*}\right)^{*} \sqsubset \partial^{F e n} k$.

Proof. Assertions a) and b) are not difficult to prove and the implications " $\Rightarrow$ " in c) and d) are direct consequences of the Brondsted-Rockafellar theorem [8]. So let us prove the converse ones. Fix any $x \in \operatorname{dom} \partial\left(h_{X}^{* *}\right)$ and any $x^{*} \in \operatorname{dom} \partial\left(k_{X}^{*}\right)^{*}$ (since these sets are nonempty by hypothesis). Then, by assumption,

$$
\partial^{\text {Fen }} h(x) \neq \varnothing \quad \text { and } \quad \partial^{F e n} k\left(x^{*}\right) \neq \varnothing
$$

and hence $h(x)=h_{X}^{* *}(x)$ and $k\left(x^{*}\right)=\left(k_{X}^{*}\right)\left(x^{*}\right)$ by assertions a) and b). Therefore, it follows, from Lemma 3.1, that $h_{X}^{* *} \geq h$ and $\left(k_{X}^{*}\right)^{*} \geq k$. By the Fenchel inequality, we get $h=h_{X}^{* *}$ and $k=\left(k_{X}^{*}\right)^{*}$ and hence $h$ and $k$ are convex.

3.3. Lemma. Let $g: X \rightarrow \mathbb{R} \cup\{+\infty\}$ and $h: X^{*} \rightarrow \mathbb{R} \cup\{+\infty\}$ be proper lower semicontinuous functions and $f: X \rightarrow \mathbb{R} \cup\{+\infty\}$ be a proper lower semicontinuous convex function. Then

a) $f=g+$ Const $\Leftrightarrow \partial f \sqsubset \partial^{\text {Fen }} g$;

b) $f^{*}=h+$ Const $\Leftrightarrow \partial^{*} f^{*} \sqsubset \partial^{\text {Fen } h}$.

Proof. a) The implication " $\Rightarrow$ " being obvious, let us prove the reverse one. The proof is heavily inspired by techniques in Rockafellar [16] where both functions are assumed to be convex. Set $j=1 / 2\|\cdot\|^{2}$. As $j$ is continuous and as $\partial f \sqsubset \partial^{\text {Fen } g}$ (by assumption), we have

$$
\partial(f+j)=\partial f+\partial j \sqsubset \partial^{F e n} g+\partial j \sqsubset \partial^{F e n}(g+j)
$$

and hence

$$
\partial^{*}(f+j)^{*} \sqsubset \partial^{*}(g+j)^{*} .
$$

As $(f+j)^{*}=f^{*} \nabla j^{*}$ is finite and continuous on $X^{*}$ (see Moreau [13]), it follows from (1) that the lower semicontinuous convex function $(g+j)^{*}$ is also finite on all $X^{*}$, hence (see [13]) continuous on $X^{*}$. We then claim

$$
\partial(f+j)^{*} \sqsubset \partial(g+j)^{*} .
$$

Indeed let $x^{* *} \in \partial(f+j)^{*}(x)$, i.e.

$$
(f+j)^{* *}\left(x^{* *}\right)+(f+j)^{*}\left(x^{*}\right)=\left\langle x^{* *}, x^{*}\right\rangle .
$$

One knows (by Moreau [13]) that there exists a generalized sequence $\left(x_{i}\right)$ in $X$ weakly- $w^{* *}$ converging to $x^{* *}$ for which $(f+j)^{* *}\left(x^{* *}\right)=\lim _{i}(f+j)\left(x_{i}\right)$. Put $\varepsilon_{i}:=(f+j)^{*}\left(x^{*}\right)+(f+j)\left(x_{i}\right)-\left\langle x^{*}, x_{i}\right\rangle \geq 0$.

Then, $x^{*} \in \partial_{\varepsilon_{i}}(f+j)\left(x_{i}\right)$ and $\varepsilon_{i} \rightarrow 0$ by (3). By the Brondsted-Rockafellar theorem, there exists $\left(z_{i}, z_{i}^{*}\right) \in \partial(f+j)$ satisfying $\left\|z_{i}-x_{i}\right\| \leq \sqrt{\varepsilon_{i}},\left\|z_{i}^{*}-x^{*}\right\| \leq \sqrt{\varepsilon_{i}}$. Then $z_{i} \stackrel{w^{* *}}{\rightarrow} x^{* *}, z_{i}^{*} \stackrel{\|\cdot\|}{\rightarrow} x^{*}$ and, for $i \geq i_{0}, z_{i} \in \partial(f+j)^{*}\left(z_{i}^{*}\right) \sqsubset \gamma \mathbb{B}_{X}$, where $\mathbb{B}_{X}$ denotes the closed unit ball of $X$ (since $(f+j)^{*}$ is locally Lipschitz). Then the 
subnet $\left(z_{i}\right)_{i>i_{0}}$ is bounded in norm and it is not difficult to see that this boundedness and relation (1) ensure $x^{* *} \in \partial(g+j)^{*}\left(x^{*}\right)$. So the claim is proved. Now, it is easy to see that (2) and the locally Lipschitzian behavior of $(f+j)^{*}$ and $(g+j)^{*}$ ensure

$$
(f+j)^{*}=(g+j)^{*}+\text { Const. }
$$

So

$$
f+j=(f+j)_{X}^{* *}=(g+j)_{X}^{* *}+\text { Const }
$$

and hence (taking the assumption into account)

$$
\partial(g+j)_{X}^{* *}=\partial(f+j) \sqsubset \partial^{F e n}(g+j)
$$

which ensures by Lemma 3.2 and (4)

$$
g+j=(g+j)_{X}^{* *}=f+j+\text { Const, }
$$

that is, $f=g+$ Const and assertion a) is proved.

b) Let us prove the implication " $\Leftarrow$ " of assertion b). It is easily seen that the assumption $\partial^{*} f^{*} \sqsubset \partial^{F e n} h$ ensures $\partial f \sqsubset \partial\left(h_{X}^{*}\right)$ (since one can verify that $\left.x \in \partial^{\text {Fen }} h\left(x^{*}\right) \Rightarrow x^{*} \in \partial\left(h_{X}^{*}\right)(x)\right)$. Then by assertion a), one has $f=h_{X}^{*}+$ Const and hence $f^{*}=\left(h_{X}^{*}\right)+$ Const. Therefore $f^{*}=h+$ Const since $\left(h_{X}^{*}\right)^{*}=h$ by the above inclusion and Lemma 3.2. So the proof of the lemma is complete.

Remark. We refer the reader to [19] where the general perturbation inclusion

$$
\partial f(x) \sqsubset \partial g(x)+\varepsilon \mathbb{B}_{X^{*}}
$$

is considered.

\section{REFERENCES}

1. H. Attouch, Convergence de fonctions convexes, des sous différentiels et semigroupes associés, C. R. Acad. Sci. Paris Sér. I Math. 284 (1977), 539-542. MR 57:13587

2. _ Variational Convergence for Functions and Operators, Pitman, Boston-LondonMelbourne (1984). MR 86f: 49002

3. H. Attouch and G. Beer, On the convergence of subdifferentials of convex functions, Archiv. Math. 60 (1993), 389-400. MR 94b:49018

4. H. Attouch and A. Damlamian, Strong solutions for parabolic variational inequalities, Nonlinear Anal. Th. Meth. Appl. 2 (1978), 329-353. MR 80a:47093

5. G. Beer, The slice topology: A viable alternative to Mosco convergence in nonreflexive spaces, Nonlinear Anal. Th. Meth. Appl. 19 (1992), 271-290. MR 93h:49025

6. - Topologies on Closed and Closed Convex Sets, Kluwer Academic Publishers, Dordrecht-Boston-London (1993). MR 95k:49001

7. G. Beer and J. Borwein, Mosco convergence and reflexivity, Proc. Amer. Math. Soc. 109 (1990), 427-436. MR 91c:46016

8. A. Brondsted and R. T. Rockafellar, On the subdifferentiability of convex functions, Proc. Amer. Math. Soc. 16 (1965), 605-611. MR 31:2361

9. C. Combari and L. Thibault, On the convergence of approximate subdifferentials of convex functions, to appear.

10. B. Lemaire, Coupling optimization methods and variational convergence, in Trends in Mathematical Optimization, series of Num. Math. Vol. 84, K. H. Hoffmann, J.-B. Hiriart-Urruty, C. Lemarechal and J. Zowe, eds., 163-179, Basel-Boston (1988). MR 91b:90154

11. A. B. Levy, R. A. Poliquin and L. Thibault, Partial extension of Attouch's theorem with applications to proto-derivatives of subgradient mappings, Trans. Amer. Math. Soc. 347 (1995), 1269-1294. MR 95k:49035

12. M. Matzeu, Su un tipo di continuità dell' operatore subdifferenziale, Boll. U.M.I. 11 (1975), 107-124. MR 57:1220

13. J.-J. Moreau, Fonctionelles convexes, mimeographed lecture notes, Collège de France, 1967. 
14. U. Mosco, On the continuity of the Young-Fenchel transform, J. Math. Anal. Appl. 35 (1971), 518-535. MR 44:817

15. R. A. Poliquin, An extension of Attouch's theorem and its applications to epi-differentiation of convexly composite functions, Trans. Amer. Math. Soc. 332 (1992), 861-874. MR 93a:49013

16. R. T. Rockafellar, On the maximal monotonicity of subdifferential mappings, Pacific J. Math. 33 (1970), 209-216. MR 41:7432

17. _ Characterization of the subdifferentials of convex functions, Pacific J. Math. 17 (1966), 497-510. MR 33:1769

18. - Generalized second derivatives of convex functions and saddle functions, Trans. Amer. Math. Soc. 322 (1990), 851-877. MR 91b:90190

19. L. Thibault and D. Zagrodny, Integration of subdifferentials of lower semicontinuous functions, J. Math. Anal. Appl. 189 (1995), 33-58. MR 95i:49032

Université Montpellier II, Laboratoire d'Analyse Convexe, Place Eugene Bataillon, 34095 Montpellier Cedex 5, France

E-mail address: thibault@math.univ-montp2.fr 